\title{
The use of narration and art in the Public Communication of Science
}

\author{
Francesca Scianitti $^{1}+$ \\ INFN Communications Office \\ Piazza dei Caprettari 70, Rome, Italy \\ E-mail: francesca.scianitti@presid.infn.it
}

Working as a laboratory for unconventional scientific communication, in the last decade the INFN Communications Office ${ }^{[1]}$ has developed innovative formats of public events, in which the performing arts are intertwined with the scientific narrative. This process was born with the goal of involving a wide variety of audiences with Particle Physics, Cosmology and Technology and increase the awareness in the research and innovation processes lead by INFN and the scientific community as a whole. The underlying idea is that a narrative approach in public events, accompanied by experiences solicited by the artistic language, can help the audience to unveil connections in the interpretation of reality, thus opening unexpected scenarios for the acquisition of knowledge. If well designed and build on verified content, these formats can offer tactics to properly engage non-expert audiences ${ }^{[2]}$.

Case studies of innovative formats are presented, as "Cosmic Tale", a conference-show about the universe birth and evolution, offered to more than 6000 people in the period from 2016 to 2019 (7 live replicas). The narrative structure of Cosmic Tale is based on the intertwining of dialogues, videos, readings and music, and moves along a well-defined storyboard, supported also by a cartoon story. More recently, the INFN and the Italian Space Agency (ASI) experimented with a format based on monologues about the same story - the first detection of gravitational waves from the coalescence of neutron stars - told from three different points of view (space satellites, terrestrial telescopes and gravitational wave detectors) and interlaced with jazz music played by professional and well-known musicians. If consciously integrated into the narrative, artistic performances can represent an unconventional voice about science, which involve artists, scientists and the public in the definition of a common storytelling and, ultimately, of a new multicultural language. Critical aspects of the conference-show formats are also presented in order to keep the discussion open on how they can be evaluated and aligned to societal needs and expectations.

European Physical Society Conference on High Energy Physics - EPS-HEP2019 -

10-17 July, 2019

Ghent, Belgium

\footnotetext{
${ }^{1}$ Speaker.

$t_{\text {on behalf of INFN Communications Office }}$
} 


\section{Reasons to innovate the way we do public communication of science}

The public communication of science is aimed at building a bridge between science and society, not simply to increase the reputation of the single research institution or to inform people, but also to enhance public awareness and appreciation of the goals and methods of scientific research as a whole. Research in science communication has increasingly confirmed, in recent years, the passage of the objective of communication from the "public understanding of science" to the "public engagement with science and technology" ${ }^{[3]}$. This doesn't mean a simple change of words and stands that communication has no longer to be conceived as a top-down process going from scientists to the public, but as a process to be designed and built up in synergy with the public. The goal is no longer to feed up people with new information and fill up their deficit of knowledge, but to "co-create" knowledge and build up a shared culture. The main difficulties in achieving these objectives are due to the fact that internal scientific credibility is normally independent of the public perception of science, of its objectives and benefits. The scientific community is not always motivated to really engage the public with science and some scientists still feel the need to simply pass on their knowledge as an asset to be shared.

A way to go over this lack can be putting scientists at the center of the scene, asking them to interpret their own story. This has been proposed as a major effort in experimenting innovative communication formats, which has become part and parcel of the INFN Communication Office activities in the last ten years. In the frame of the conference-show format, in particular, the scientist knows that he is giving to the public a story with a narrative structure, in which art is also an important voice in the storytelling and tells the same story with its own language. This is something very far from a formal approach to science communication. When scientists are well conscious of their role in this context, their communication can be more integrated with the whole story and a closer relationship with the public ca be established.

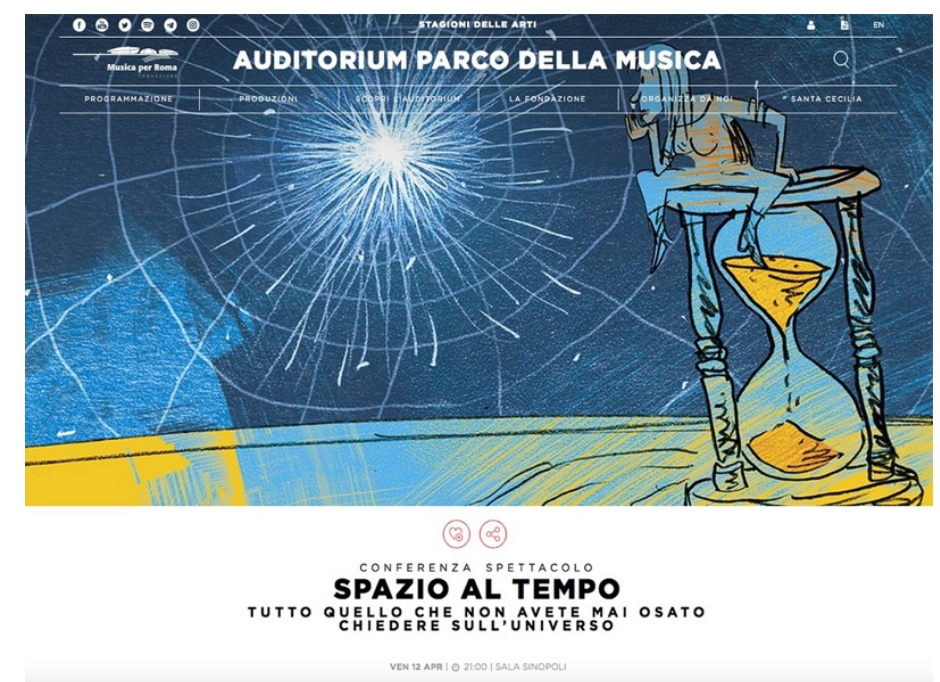

Fig. 1 The conference-show "Space to Time", staged in 2019 for the National Geographic Science Festival in Rome. The storyboard was based on a sequential story of the universe evolution, with the contribution of readings and live music. 


\subsection{Why using narratives and art to talk about science}

The INFN Communications Office started to use narratives and the artistic language to communicate science in the frame of innovative formats of public events, which have been named conference-shows. These have been designed since the years 2011 to communicate physics and INFN research goals to a variety of non-expert audiences, running from students to adults and families. The format is based on the intertwining among storytelling, performances, videos, animations, and monologues or dialogues among scientists. Although the experience isn't still based on a rich literature of theoretical issues in science communication, nor on well-structured best practices and experimentations, results in terms of public satisfaction are very encouraging.

Since 2011, we have been experiencing different formats of public events all around Italy. The number of events has increased during the last years and this was possible thanks to specific agreements with theaters and other venues, aimed at co-producing the events, thus making them more sustainable under the economical and organisational points of views. Today, eight years after the beginning of this experience, the INFN Communications Office is still on a way of experimenting with new formats, aiming at defining the best narrative structure, language and tools to share contents and stories with our audiences.

\subsection{The conference-show format}

Although the way we use narratives and artistic performances to build the structure of science events has a purely empirical base, it finds a theoretical soil on the existing literature in the field of science education. According to Bruner's thought ${ }^{[4]}$, in particular, narrative is intrinsically connected with the process of meaning creation and aims to provide a reasonable representation of individual experiences, thus having the power to solicit a privileged status in human cognition: narrative reports to the self-construction of contents, improving understanding and learning. On the other hand, art is a proficient language to talk about human experiences and human senses: it can involve both sight and hearing in an unconventional and not passive acceptance of contents, thus stimulating parallel insights into ideas. Art and narrative, together, thus have the potential to reveal otherwise invisible connections in reality, offering the public the opportunity for greater understanding, interest and involvement.

\section{Designing science conference-shows}

How can we design the structure of a conference-show? As a first step, the reference frame where the event is inserted has to be investigated. This step has an influence on the different choices we have to make: first of all, the target we want to reach, then the context where the communication is realized and, finally, the message we want to pass. These three aspects can be synthetically called the "Who, Where, What" choices. A theatre or a museum, for example, are very different contexts for public events, engaging different targets and fulfilling at different requirements. Once these choices have been made, the storyboard skeleton has to be designed, taking care to make it logicalscientific, solid and well-verified in its contents. 
Afterwards, a narrative structure will be superposed to this skeleton, reflecting the standard linear narrative scheme commonly divided into preamble, crisis and solution. This is not mandatory, but it's usually the easiest narrative structure to be used, capable to give rhythm to the story and to organize its contents in a linear sequence, avoiding confusing repetitions or conceptual gaps. The whole story arises from the overlap of the two structures, the logic-scientific storyboard and the narrative one, and from the intertwining of monologues or dialogues, images and videos, artistic performances like music, live-drawings, readings and so on. However, improvisations by scientists and artists have a fundamental role and necessarily turns around this well-defined scientific and narrative sequence.
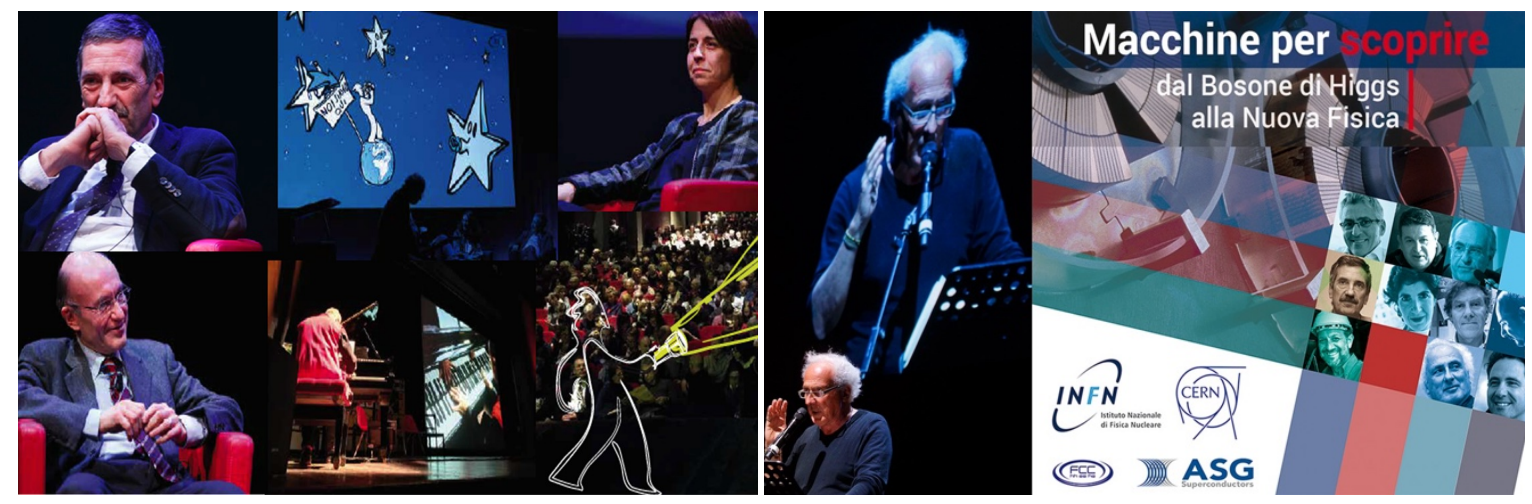

Fig. 1 Left image: the conference-show "Cosmic Tale" was staged seven times in the years between 2016 and 2019, reaching about 6000 people. Right image: Discovery Machines. From the Higgs Boson to New Physics", an institutional event about the Future Circular Collider (FCC) project for a future collider at CERN, was staged at the Auditorium Parco della Musica in Rome for an audience of 1200 people (in English: https://youtu.be/ BKx0qVyHmBs).

\subsection{Cosmic Tale: a case of success}

Among all the events realized by the INFN Communications Office in recent years, "Cosmic Tale" is certainly the most successful one, with 7 replicas in Italy from 2016 to 2019, touching different towns, from the biggest ones, as Rome, Turin, Bergamo and Genoa, to very small towns in the countryside of Northern and Central Italy. In all these cases, replicas have been proposed and produced by the host theaters.

In Cosmic Tale, the dialogue among scientists turns around three animated cartoons, and intertwines with readings and piano jazz music. The storyboard relies on a well-designed sequence of scientific ideas and is supported by three thematic animated cartoons: the dialogue thus becomes more linear, sequential and effective. Since the first edition in Rome, at the Auditorium Parco della Musica, main actors have been the physicists Viviana Fafone, Fernando Ferroni, Antonio Masiero. Artistic contributions have been interpreted by the jazz pianist Umberto Petrin, the actress Maria Giulia Scarcella, the illustrator Luca Ralli and the well-known conductor Neri Marcorè.

Quantitative assessments have not yet been made on this event and this will be a further step in implementing it. However, we are encouraged by the qualitative satisfaction that we recorded from the public, which we believe is mainly due to the narrative structure and its linearity, which makes contents comprehensible at different levels of attitudes and knowledge. 


\subsection{A well-defined structure to support improvisation}

Three cartoons on Space, Time and Gravity represent the skeleton of Cosmic Tale's narrative structure and help to organize the storyboard in three acts, along the conventional structure consisting of a premise, a crisis and a solution. The premise is given by a first observation of what surrounds us and a subsequent simple question: where are we in space and how do we get to know the universe? Our knowledge is based on astronomical, cosmological and particle physics observations that describe a visible universe made of stars and particles, which we know in enough detail (fundamental issues in this first act are the Standard Model and the Higgs Boson's discovery). This comforting statement is followed by the "crisis": there are questions in Physics that we cannot answer. They tell about a dark universe that we do not know (with particular attention to dark matter), to study which the scientific community designs and carries out highly performing and challenging experiments and detectors (with particular attention to CERN and INFN Gran Sasso National Laboratories). Finally, the resolving situation is given by something new that comes to shed light on this main obscure part of the universe. The discovery of gravitational waves, in particular, (with a major focus on the LIGO-VIRGO collaborations and to space and earth telescopes) offered a new look to our universe, restoring a new image of it.

Visual and artistic narratives, made of videos, cartoons, jazz piano music and readings (extracts from Dino Buzzati and Bill Bryson), are superimposed to this structure. In this context, scientists experts in cosmology, high-energy physics and gravitational waves - tell a well-defined story without improvisation being prevented. Scientists outline their answers at the right moment and can insert anecdotes or new ideas, but they know that everything relies on a narrative structure, a welldefined sequence that has to be respected and asks to stay on the rails.

\subsection{An institutional framework for a general public addressed event}

Staged at the Auditorium Parco della Musica in Rome in 2016, "Discovery Machines" is an example of an institutional event, the framework of which had numerous constraints. The context was that of the FCC Week 2016 conference in Rome, which revolved around the Future Circular Collider project at CERN. The event was offered to citizens and the audience was therefore composed of a minimal part of conference attendees and a relevant part made of general public. The first constraint to take in consideration in designing this public event was the very technical subject, which did not really fit with a target made of nonexperts. A second important constraint was given by the number of people involved as speakers, all with relevant roles and rich contents to talk about. Taking care of all these aspects required the use of a rigid narrative structure in which each topic and scientist had a specific and precise role. Authoritative speakers were the physicists Fabiola Gianotti, Fernando Ferroni, Antonio Zoccoli, Sergio Bertolucci, Guido Tonelli, Michelangelo Mangano and the economist Massimo Florio. Artistic contributions were given by the wellknown writer Stefano Benni, the jazz musician Umberto Petrin and the illustrator Luca Ralli. The contributions of jazz music and literature, with readings of extracts from Borges and Queneau, helped to give rhythm and underline the passages in the story. The event has been simultaneously translated into English for non-Italian participants and for the researchers connected via streaming at CERN. 


\subsection{Talking about discoveries}

More recently, the INFN and the Italian Space Agency (ASI) experimented with a new format based on monologues given by three scientists who told the same story - that of the direct detection of the coalescence of neutron stars - from three different points of view (space satellites, terrestrial telescopes and gravitational wave detectors). In "Lights \& Waves Rhapsody" jazz played a dominant role, giving rhythm to the speech and underlining suspensions and climaxes, taking sometimes the scene to create a suitable environment for the public perception of ideas, as if the music staged its own monologue. The story was written in agreement with the three scientists, in order to guarantee an absolute formal propriety of contents, and then integrated to adhere to a unique coherent style. The personal interpretation from the protagonists of the discovery was able to create a great emotional impact on the public, also enhanced by the jazz music performances played by three famous jazz musicians: Danilo Rea, Michele Rabbia, Paolo Damiani. Main actors of "Lights \& Waves Rhapsody" were the scientists Fulvio Ricci (INFN), Paolo D'Avanzo (INAF) and Immacolata Donnarumma (ASI).

When conceived appropriately and evaluated in its impact on the audience, this kind of scientific narratives can open unprecedented synthesis windows between science and art, increasing the engagement of the public and giving rise to unexpected insights on abstract concepts of physics and the way research succeeds in discoveries.

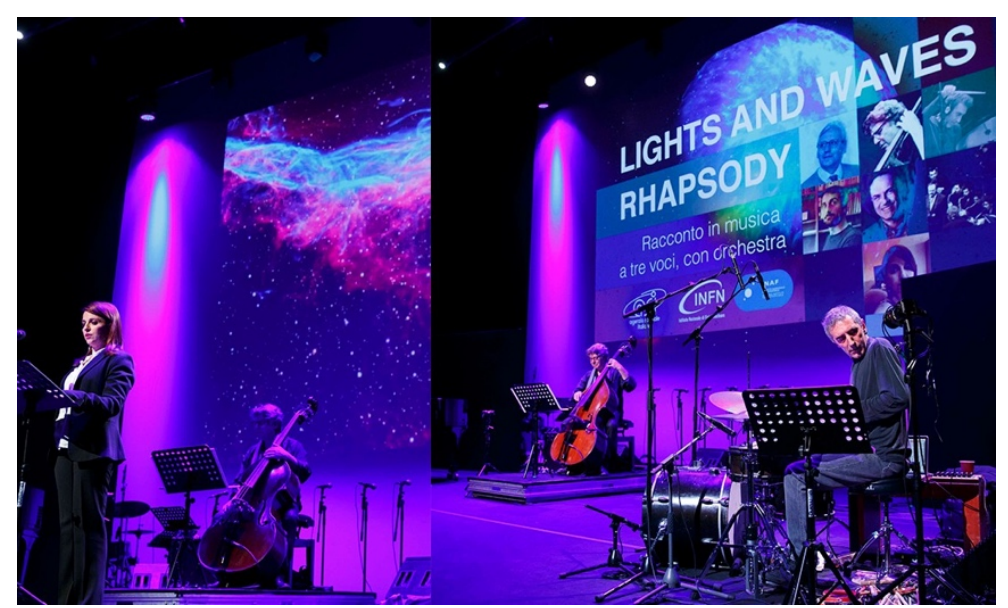

Fig. 3 "Lights and Waves Rhapsody" is a show-conference based on jazz music and monologues by scientists. The story is interpreted by three scientists directly involved in the first direct detection of neutron star coalescence, obtained with the choral contribution of different detectors: gravitational wave interferometers, electromagnetic ground detectors and space satellites.

\section{Next step: evaluation and quality in science communication through public events}

The improvement of quality practices through evaluation will be our next step to identify the best way to involve the public and strengthen the quality of their experience. The first simple feedback we could record so far in our experiences is given by the large number of people attending the events and a high specific interest has been recorded in the case of the "Cosmic Tale" show-conference, which has been self-produced by some theaters. This is also due to an undoubted growing interest in topics related to physics and cosmology in recent years. 
A quantitative assessment is required in order to implement the conformity of our interventions with the expected benefits on the public. Creating opportunities for citizens science and encouraging capacity building in the public are among the main objectives of INFN Third Mission, also taking into account ethical aspects such as the respect for diversity, gender equality, health and safety and the re-launching an idea of research as a societal effort and a common capital. A further step will be the growing alignment of INFN public engagement actions and message with the strategic goals of INFN research.

\section{References}

[1] Francesca Scianitti et al., The role of images in the storytelling of the invisible, EPS-HEP 2017, European Physical Society Conference on High Energy Physics, 5-12 July 2017, DOI: $10.22323 / 1.314 .0568$

[2] Dahlstrom, Michael F. Using narratives and storytelling to communicate science with non expert audiences, PNAS vol 111 suppl. 4 September 16, 2014 [www.pnas.org/cgi/doi/10.1073/pnas. 1320645111]

[3] Irwin, A. The Politics of Talk: Coming to Terms with the 'New'Scientific Governance, Social Studies of Science 36(2): 299-320

[4] Bruner, J. S. Actual minds. Possible words, Harvard University Press, Cambridge, 1986 